\title{
LKB1 promotes radioresistance in esophageal cancer cells exposed to radiation, by suppression of apoptosis and activation of autophagy via the AMPK pathway
}

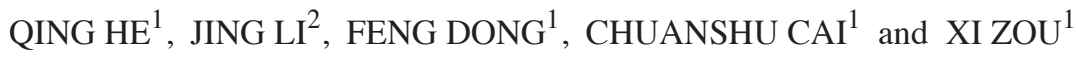 \\ Departments of ${ }^{1}$ Radiotherapy and ${ }^{2}$ Radiology, The First Affiliated Hospital of Fujian Medical University, \\ Fuzhou, Fujian 350005, P.R. China
}

Received May 19, 2016; Accepted April 5, 2017

DOI: $10.3892 / \mathrm{mmr} .2017 .6852$

\begin{abstract}
Liver kinase B (LKB) 1 acts as a tumor suppressor in a broad spectrum of human cancers, and is important in chemoradiotherapy treatment of various tumor types. However, the potential function of LKB1 in esophageal cancer radiotherapy remains to be elucidated. The aim of the present study was to investigate the role of LKB1 in radiosensitivity of esophageal cancer in vivo and in vitro, and to explore its molecular mechanism. Eca-109 cells transfected with LKB1 overexpression plasmid were xenografted into nude mice and subjected to irradiation and it was observed that the tumor volume was significantly increased in LKB1-overexpressed tumors compared with that of the control tumors. The in vitro study revealed that LKB1 overexpression led to the radioresistance of Eca-109 cells, as determined by MTT and colony formation assays. Furthermore, it was demonstrated that LKB1 overexpression inhibited apoptosis and activated autophagy of Eca-109 cells following radiation treatment, as determined by flow cytometry and western blot analyses. AMP-activated protein kinase (AMPK) inhibition attenuated LKB1-induced radioresistance of Eca-109 cells. To the best of our knowledge, the present study, for the first time, confirmed that LKB1 induces radioresistance of esophageal cancer cells to irradiation via suppression of apoptosis and activation of autophagy, and AMPK mediates this function of LKB1 in esophageal cancer radiotherapy. These findings suggest that LKB1 may act as a novel target in the future, to maximize the efficiency of esophageal cancer radiotherapy.
\end{abstract}

Correspondence to: Dr Jing Li, Department of Radiology, The First Affiliated Hospital of Fujian Medical University, 20 Chazhong Road, Fuzhou, Fujian 350005, P.R. China

E-mail: li_jing72@sina.cn

Key words: liver kinase B1, radioresistance, esophageal cancer, apoptosis, autophagy, AMP-activated protein kinase

\section{Introduction}

Esophageal cancer is one of the most frequently diagnosed cancers and is responsible for numerous cancer-associated mortalities worldwide (1), with an increasing incidence annually (2). Esophageal cancer has two predominant histological types: esophageal squamous cell carcinoma (ESCC), which accounts for 95\% of all esophageal cancers in China, and esophageal adenocarcinoma, which frequently occurs in developed countries (1).

Radiotherapy is an essential therapeutic method in the treatment of patients with inoperable and locally advanced ESCC. However, the response of esophageal cancer to radiotherapy is variable, and the majority of ESCC patients do not benefit from radiotherapy due to radioresistance (3-6). Therefore, searching for molecular markers which may enhance the radiosensitivity of esophageal cancer is of primary concern, in order to improve clinical outcomes.

Liver kinase B (LKB) 1, additionally termed, serine/threonine kinase 11, is important in various biological processes, including cell growth, apoptosis and DNA damage response, cell motility, energy metabolism and cell polarity $(7,8)$. Mutations in the LKB1 gene have been associated with a broad spectrum of human cancers, and LKB1 has been indicated to be a critical tumor suppressor $(9,10)$. Recently, various studies have suggested that LKB1 is involved in the regulation of radiosensitivity of cancer cells, and may be important in regulating the radiosensitivity of different tumor types $(11,12)$. However, the potential function of LKB1 in esophageal cancer radiotherapy remains to be elucidated.

The present study investigated the role of LKB1 in the radiosensitivity of esophageal cancer and its molecular mechanism. The results may aid in providing a novel mechanism to improve the efficacy of radiotherapy in the treatment of esophageal cancer patients in the future.

\section{Materials and methods}

Animal experiments. All animal experiments were approved by the Ethics Committee of The First Affiliated Hospital of Fujian Medical University (Fujian, China). A total of 156 male BALB/C nude mice, (age, 5-6 weeks; weight, 16-20 g) were 
provided by the Shanghai Laboratory Animal Research Center (Shanghai, China). The mice were maintained in individual cages at a controlled temperature $\left(22 \pm 2^{\circ} \mathrm{C}\right)$ and humidity $(55 \%)$, under $12 / 12 \mathrm{~h}$ light/dark cycles, with free access to food and water. To develop xenograft tumors, Eca-109 cells transfected with LKB1 overexpression plasmid or empty vector were harvested, washed with PBS and implanted into the hind limb of the BALB/C nude mice $\left(2 \times 10^{6}\right.$ cells $\left./ 0.1 \mathrm{ml}\right)$. When xenograft tumors reached a mean diameter of $5 \mathrm{~mm}$, the 'radiation group' animals were irradiated every 4 days with a dose of 12 Gy radiation in three fractions. Tumor growth was measured every 2 days by a caliper until day 25 , and the tumor volume $\left(\mathrm{mm}^{3}\right)$ was calculated according to the formula: Tumor volume $\left(\mathrm{mm}^{3}\right)=$ length $(\mathrm{mm}) \mathrm{x}$ width $\left(\mathrm{mm}^{2}\right) / 2$.

Cell culture. Eca-109 cells were obtained from the Cell Bank of the Chinese Academy of Sciences (Shanghai, China). The cells were maintained in Dulbecco's modified Eagle's medium (Hyclone; GE Healthcare, Logan, UT, USA) supplemented with $10 \%$ fetal bovine serum (Gibco; Thermo Fisher Scientific, Inc., Waltham, MA, USA) and $100 \mu \mathrm{g} / \mathrm{ml}$ penicillin at $37^{\circ} \mathrm{C}$ in a humidified atmosphere containing $5 \% \mathrm{CO}_{2}$. Compound $\mathrm{C}$ was purchased from EMD Millipore (Billerica, MA, USA) and used at a concentration of $20 \mu \mathrm{M}$.

Cell transfection. The LKB1-pcDNA3.1 plasmid was synthesized by Shenzhen Zhonghong Boyuan Biological Technology Co., Ltd. (Shenzhen, China). A total of $1 \mu \mathrm{g}$ plasmid DNA was transfected into the Eca-109 cells using Lipofectamine 2000 (Invitrogen; Thermo Fisher Scientific, Inc.) according to the manufacturer's protocol.

MTT assay. Cell viability was determined via an MTT assay. The cells were plated in 96-well plates at a density of $5 \times 10^{4}$ cells/well. Following incubation for 1, 2, 3 and 4 days, $5 \mu 1$ of MTT solution (1 mg/ml; Sigma-Aldrich; Merck KGaA, Darmstadt, Germany) was added to each well, and the plates were incubated for an additional $4 \mathrm{~h}$. Finally, dimethyl sulfoxide (Sigma-Aldrich; Merck KGaA) was added to dissolve the formazan crystals and the absorbance was measured at a wavelength of $570 \mathrm{~nm}$.

Colony formation assay. Eca-109 cells were seeded into six-wells plates, at a density of 500 cells/well. Following overnight culture at $37^{\circ} \mathrm{C}$, the cells were exposed to radiation at 0 , 2, 4, 6 and 8 Gy with an average dose rate of $100 \mathrm{cGy} / \mathrm{min}$. Following incubation at $37^{\circ} \mathrm{C}$ in an environment containing $5 \% \mathrm{CO}_{2}$ for 10 days, the colonies were fixed in $4 \%$ formaldehyde at room temperature for $30 \mathrm{~min}$, and stained with crystal violet at room temperature for $2 \mathrm{~h}$. Stained cells were observed under a microscope (TS100; Nikon Corporation, Tokyo, Japan) and colonies containing $>50$ cells were counted as clonogenic survivors.

Flow cytometry. The Annexin V-FITC and Propidium Iodide (PI) Apoptosis assay kit (Nanjing KeyGen Biotech Co., Ltd., Nanjing, China) was used to determine the percentage of cells undergoing apoptosis, according to the manufacturer's protocol. Briefly, cells were harvested $8 \mathrm{~h}$ post-irradiation at a dose of 8 Gy. Following washing with PBS, $1 \times 10^{6}$ cells were dual-stained with $10 \mu \mathrm{l}$ PI and $5 \mu \mathrm{l}$ Alexa Fluor 488-Annexin V at room temperature for $15 \mathrm{~min}$, and subsequently analyzed by flow cytometry (BD FACSCanto II flow cytometer; BD Biosciences, Franklin Lakes, NJ, USA) using the BD FACSCanto $^{\mathrm{TM}}$ Clinical software version 2.1 (BD Biosciences).

Western blot analysis. Cells were lysed in a radioimmunoprecipitation assay lysis buffer (Beyotime Institute of Biotechnology, Shanghai, China) at room temperature for $5 \mathrm{~min}$. Cell lysates were centrifuged at $13,000 \mathrm{x} \mathrm{g}$ for $5 \mathrm{~min}$ at room temperature, and the supernatants were harvested. Protein concentrations were quantified using a BCA kit (Pierce; Thermo Fisher Scientific, Inc.). Equal amounts of extracted protein samples $(5 \mu \mathrm{g})$ were separated by $12 \%$ SDS-PAGE and transferred onto polyvinylidence difluoride membranes (EMD Millipore). The membranes were blocked with $3 \%$ bovine serum albumin (Sangon Biotech Co., Ltd., Shanghai, China) at $4^{\circ} \mathrm{C}$ overnight, and the blots were probed with rabbit polyclonal antibodies against LKB1 (catalog no. ab79355; $1: 500)$, rabbit polyclonal to active caspase-3 (catalog no. ab2302; 1:200), mouse monoclonal to cleaved poly(ADP-ribose) polymerase (PARP; catalog no. ab13907; 1:200), rabbit polyclonal to microtubule-associated protein 1 light chain $3 \alpha / \beta$ (LC3 $\alpha / \beta$; catalog no. ab128025; 1:800) (all from Abcam, Cambridge, MA, USA), rabbit monoclonal to phosphorylated (p)-AMP-activated protein kinase (AMPK) $\alpha$ (Thr172; catalog no. 4188; 1:400), and mouse monoclonal to beclin-1 (catalog no. 4122; 1:400) (both from Cell Signaling Technology, Inc., Danvers, MA, USA) and mouse monoclonal to GAPDH (catalog no. ab8245; 1:2,000; Abcam) at $37^{\circ} \mathrm{C}$ for 2 h. Following washing with Tris buffered saline Tween-20, the membranes were incubated with goat anti-mouse horseradish peroxidase-conjugated immunoglobulin (Ig) G (catalog no. sc-2005; 1:5,000) or goat anti-rabbit horseradish peroxidase-conjugated IgG (catalog no. sc-2004; 1:5,000) (both from Santa Cruz Biotechnology, Inc., Dallas, TX, USA) secondary antibodies at $37^{\circ} \mathrm{C}$ for $1 \mathrm{~h}$, and protein bands were visualized by a SuperSignal West Femto kit (Pierce; Thermo Fisher Scientific, Inc.). Blots were semi-quantified by densitometric analysis using Image J software version 1.46 (National Institutes of Health, Bethesda, MD, USA).

Statistical analysis. Statistical analysis was performed using SPSS statistical software, version 19.0 (IBM SPSS, Armonk, NY, USA). Data are presented as the mean \pm standard deviation of 3 independent experiments. The statistical significance of the differences between groups was assessed using unpaired Student's t-test for pair-wise comparisons or one-way analysis of variance followed by a post hoc least significant difference test for multiple comparisons. $\mathrm{P}<0.05$ was considered to indicate a statistically significant difference.

\section{Results}

LKB1 expression is induced in Eca-109 cells following radiation treatment. To investigate the effect of radiation treatment on LKB1 expression, the Eca-109 cells were subjected to irradiation from 1-8 Gy 10 times, and then western blot analysis was performed to examine LKB1 expression level in 

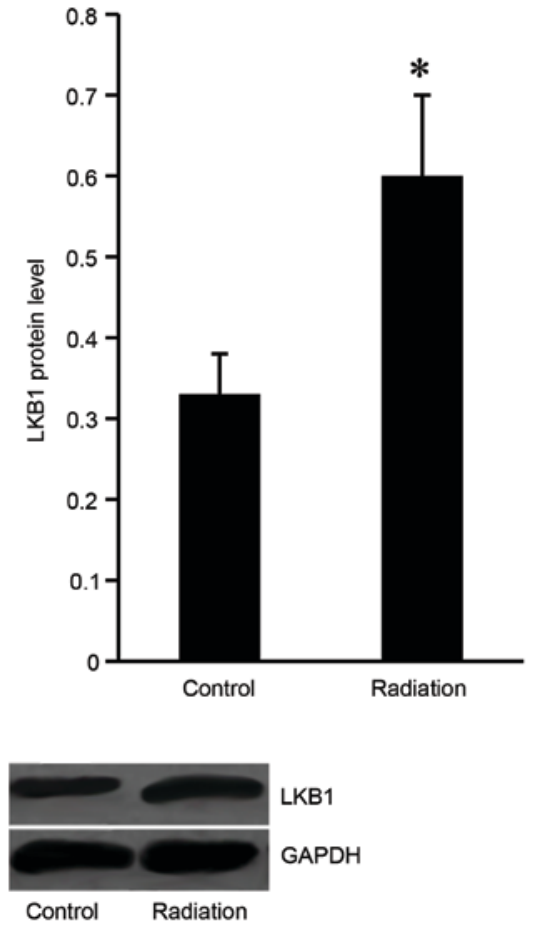

Figure 1. Expression of LKB1 protein in Eca-109 cells following radiation treatment. Representative image and quantification of LKB1 protein expression level and GAPDH control, following irradiation. ${ }^{*} \mathrm{P}<0.05$ vs. control. LKB1, liver kinase B1.
A

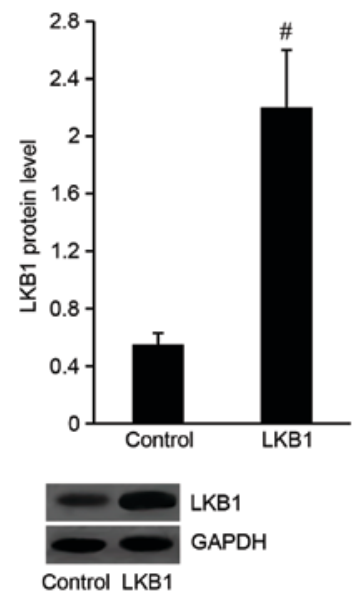

B

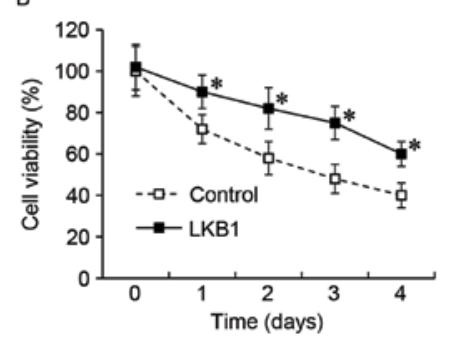

c

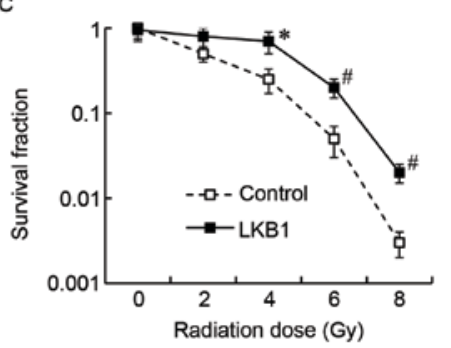

Figure 2. LKB1 modulated the radiosensitivity of Eca-109 cells. (A) Expression of LKB1 protein in Eca-109 cells following transfection with the LKB1-pcDNA3.1 plasmid. (B) Cell viability of LKB1 overexpressed-Eca-109 cells following radiation treatment. (C) Survival fraction of LKB1 overexpressed-Eca-109 cells following radiation treatment. ${ }^{*} \mathrm{P}<0.05$, ${ }^{\#} \mathrm{P}<0.01$ vs. control. LKB1, liver kinase B1.

the Eca-109 cells. The results demonstrated that LKB1 protein expression increased in the irradiated cells compared with the untreated cells (Fig. 1).

LKB1 modulates the radiosensitivity of Eca-109 cells. To determine the effect of LKB1 on the modulation of Eca-109 cell radiosensitivity, Eca-109 cells were transfected with the LKB1-pcDNA3.1 plasmid to overexpress LKB1, and the pcDNA3.1 vector was transfected as the control. As demonstrated in Fig. 2A, the LKB1 protein level was significantly increased in LKB1-pcDNA3.1-transfected cells compared with the control.

Following irradiation at a dose of 8 Gy for $24 \mathrm{~h}$, an MTT assay was performed and the results demonstrated that the cell viability was significantly increased in the LKB1-pcDNA3.1 group compared with the control group (Fig. 2B). The results of the colony formation assay demonstrated that the LKB1-pcDNA3.1-transfected cells exhibited an increased survival fraction compared with control cells (Fig. 2C).

LKB1 induces radioresistance in xenograft tumor models. To investigate if LKB1 affected the radiosensitivity of esophageal cancer in vivo, xenograft tumors with a high level of LKB1 expression were established in BALB/c nude mice and exposed to irradiation. Xenograft tumors transduced with empty vector were used as a control. It was observed that the tumor volume was significantly increased in LKB1-overexpressed tumors compared with that of the control tumors, when treated with radiation (Fig. 3).

LKB1 inhibits apoptosis and activates autophagy of Eca-109 cells following radiation treatment. Subsequently, the present study explored the role of LKB1 in apoptosis and autophagy of Eca-109 cells following radiation. Following exposure to radiation at $8 \mathrm{~Gy}$ for $24 \mathrm{~h}$, the cells were harvested for flow cytometry and western blot assays. The results of the flow cytometry assay demonstrated that the cell apoptosis rate was significantly downregulated in LKB1-pcDNA3.1-transfected cells compared with the control cells (Fig. 4A). In addition, the expression of apoptotic proteins, including the active caspase-3 and cleaved PARP-1 were markedly reduced in LKB1-pcDNA3.1-transfected cells compared with control cells. The expression levels of autophagy-associated proteins (beclin-1 and LC $3 \alpha / \beta$ ) were revealed to be significantly increased in LKB1-pcDNA3.1-transfected cells compared with control cells (Fig. 4B).

Effect of LKBI on radiosensitivity of Eca-109 cells is mediated by $A M P K$. To further examine the molecular mechanism underlying the effect of LKB1 on radiosensitivity of Eca-109 cells, the cells were transfected with the LKB1-pcDNA3.1 plasmid and/or treated with compound C, an AMPK inhibitor, and then subjected to irradiation. The activation of AMPK was measured by AMPK $\alpha$ phosphorylation on Thr172. The western blot analysis demonstrated that the expression level of p-AMPKa (Thr172) in LKB1-pcDNA3.1-transfected cells was significantly upregulated compared with pcDNA3.1-transfected cells, following radiation treatment. Compound $\mathrm{C}$ effectively inhibited p-AMPK $\alpha$ (Thr172) protein expression (Fig. 5A). As presented in e. 5B and $\mathrm{C}$, following irradiation treatment, the cell viability and survival fraction were significantly increased in LKB1-pcDNA3.1-transfected cells compared with control cells. However, following inhibition of AMPK, the increased cell viability and survival fraction induced by LKB1 was inhibited. 
A

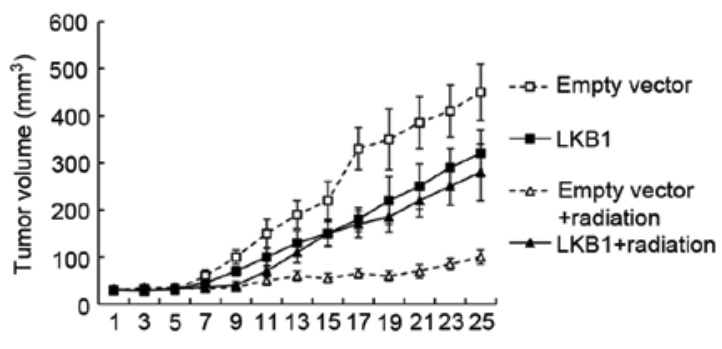

B

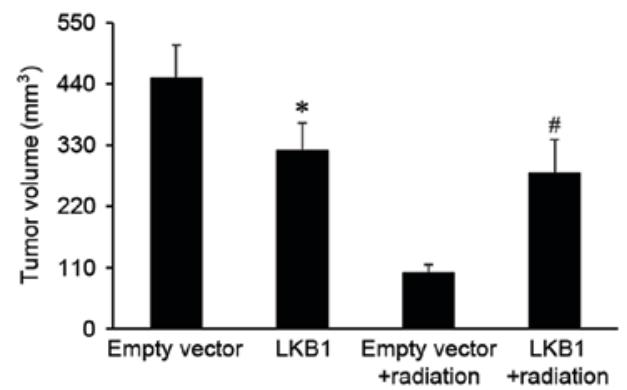

Figure 3. LKB1 induced radioresistance in xenograft tumor models. (A) The growth curves of xenograft tumors. (B) The volume of xenograft tumors on day 25 . ${ }^{2} \mathrm{P}<0.05$ vs. empty vector group; ${ }^{*} \mathrm{P}<0.01$ vs. empty vector + radiation group. LKB1, liver kinase $\mathrm{B} 1$.

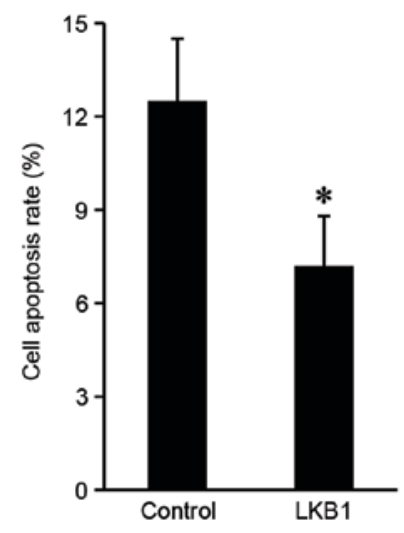

B

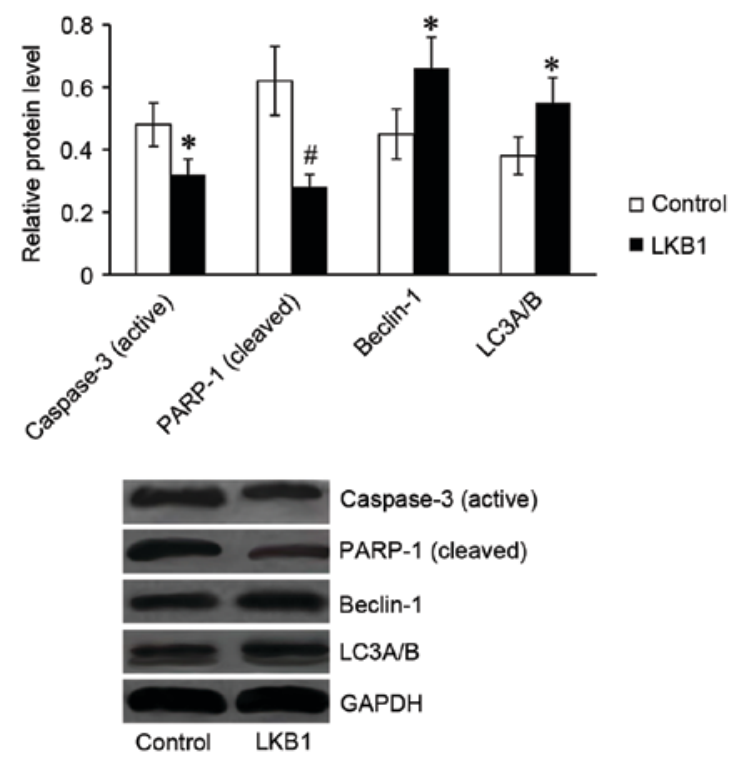

Figure 4. LKB1 inhibited apoptosis and activated autophagy of Eca-109 cells following radiation treatment. (A) Cell apoptosis rate of LKB1 overexpressed-Eca-109 cells following radiation treatment. (B) Expression of apoptotic proteins (active caspase-3 and cleaved PARP-1) and autophagy-associated proteins (Beclin-1 and LC3A/B) in LKB1 overexpressed-Eca-109 cells following radiation treatment. ${ }^{*} \mathrm{P}<0.05,{ }^{~} \mathrm{P}<0.01$ vs. control. LKB1, liver kinase B1; PARP-1, poly(ADP-ribose) polymerase 1; LC $3 \alpha / \beta$, microtubule-associated protein 1 light chain $3 \alpha / \beta$.

A
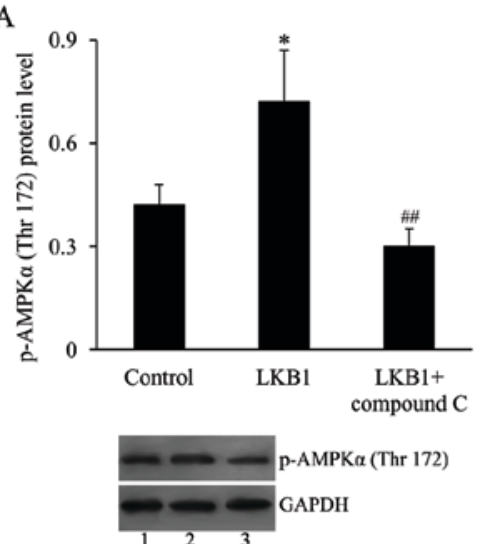

B

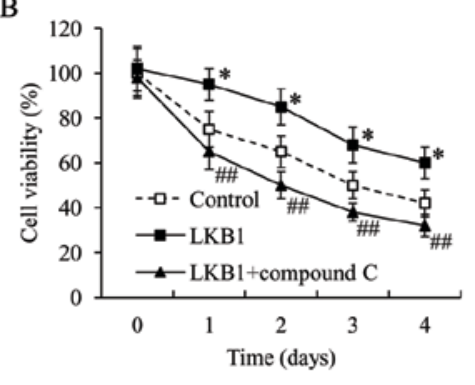

C

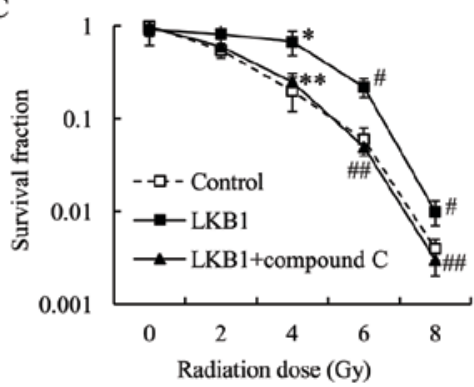

Figure 5. The effect of LKB1 on radiosensitivity of Eca-109 cells was mediated by AMPK. (A) Representative image and quantification of expression level of p-AMPK $\alpha$ (Thr172) in Eca-109 cells transfected with LKB1-pcDNA3.1 plasmid and then treated with compound C, following radiation exposure. Lane 1, control; lane 2, LKB1; lane 3, LKB1 + compound C. (B) Cell viability of Eca-109 cells transfected with LKB1-pcDNA3.1 plasmid and treated with compound C, following radiation exposure. (C) Survival fraction of Eca-109 cells transfected with LKB1-pcDNA3.1 plasmid and treated with compound C, following radiation exposure. ${ }^{*} \mathrm{P}<0.05,{ }^{\#} \mathrm{P}<0.01$ vs. control group; ${ }^{* *} \mathrm{P}<0.05$ and ${ }^{\# \#} \mathrm{P}<0.01$ vs. LKB1 group. LKB1, liver kinase B1; $\mathrm{p}$, phosphorylated; AMPK, AMP-activated protein kinase. 


\section{Discussion}

To the best of our knowledge, LKB1 appears to exhibit dual characteristics in human cancers: LKB1 acts as a well-known tumor suppressor by suppressing cell growth and metastasis, however it additionally enhances chemo and radioresistance of various tumor cells. Using genetically engineered mouse models of primary lung adenocarcinoma, it was previously demonstrated that loss of the LKB1 gene impairs the response of Kras-mutant lung cancers to standard chemotherapy (13) and renders the tumors less responsive to radiotherapy (11). Saigusa et al (12) revealed that in patients with locally advanced rectal cancer treated with pre-operative chemoradiotherapy, LKB1 gene expression levels are increased in patients with a poor pathological response and tumor recurrence, suggesting that LKB1 expression may be involved in resistance to chemoradiotherapy. In addition, Xia et al (14) demonstrated that LKB1 enhances chemoresistance in breast cancer. These reports indicate that LKB1 may serve different roles in the radiosensitivity of various types of tumor. Wang et al (15) revealed that downregulation of LKB1 is associated with esophageal cancer progression and LKB1 may inhibit esophageal cancer cell proliferation. However, few studies have reported the association between LKB1 and radiosensitivity of esophageal cancer. The present study demonstrated that LKB1 expression was significantly upregulated in Eca-109 cells in response to radiation, and it was hypothesized that LKB1 may modulate the radiation response of esophageal cancer. Following this, the in vivo and in vitro studies revealed that LKB1 overexpression suppressed the esophageal cancer cell response to irradiation, which confirmed that LKB1 induced radioresistance of esophageal cancer.

Radiation induces an apoptotic response which destroys the cells following radiotherapy (16). The intrinsic and extrinsic apoptotic pathways lead to the activation of caspases, which proteolytically cleave the substrate PARP. Autophagy is 'the second apoptosis' which is important in the programmed cell apoptotic response (17). Autophagy may be activated as a response of ionizing radiation and targeting autophagy is an antitumor strategy which is currently of interest (18-20). The present study revealed that LKB1 suppressed apoptosis and activated autophagy of esophageal cancer cells with radiotherapy. These data suggested that apoptosis and authophagy were the potential mechanisms underlying the effect of LKB1 on the induction of radioresistance in the esophageal cancer cells.

LKB1 has previously been demonstrated to regulate the activities of various signal transduction pathways, including bone morphogenetic protein receptor signaling (21) and the Notch (22) and Wnt (23) signaling pathways. LKB1 is a primary upstream kinase of AMPK, an enzyme that regulates a wide variety of cellular functions, including growth, metabolism, stress, autophagy and polarity $(24,25)$. Ionizing radiation may activate AMPK in various human cancer cells (26). It has been suggested that AMPK may be important in the cellular response to radiotherapy (27-29). To explore the potential molecular mechanism underlying the function of LKB1 in esophageal cancer radiosensitivity, the present study investigated AMPK signaling. LKB1 increases AMPK activity by phosphorylating its Thr172 residue $(30,31)$. Compound C, an AMPK inhibitor, was used to suppress AMPK activity. It was demonstrated that the promotive effect of radioresistance induced by LKB1 on Eca-109 cells, was attenuated by AMPK inhibition. These results suggested that AMPK mediates the radioresistance of Eca-109 cells via LKB1.

In conclusion, LKB1 acts as a tumor suppressor in various cancers, however it was revealed to exhibit a differing function with exposure to radiotherapy. To the best of our knowledge, the present study demonstrated for the first time, that LKB1 induced radioresistance of esophageal cancer cells to irradiation via suppression of apoptosis and activation of autophagy, and this effect was mediated by AMPK. Further studies are required to investigate the association between increased autophagy and increased radioresistance of esophageal cancer cells to further clarify the molecular mechanisms underlying these events. The findings will aid in the understanding of the occurrence of radioresistance in esophageal cancer treatment, and may provide a novel target to maximize the efficiency of esophageal cancer radiotherapy in the future.

\section{References}

1. Enzinger PC and Mayer RJ: Esophageal cancer. N Engl J Med 349: 2241-2252, 2003

2. Schuchert MJ, Luketich JD and Landreneau RJ: Management of esophageal cancer. Curr Probl Surg 47: 845-946, 2010.

3. Fareed KR, Kaye P, Soomro IN, Ilyas M, Martin S, Parsons SL and Madhusudan S: Biomarkers of response to therapy in oesophago-gastric cancer. Gut 58: 127-143, 2009.

4. Gillies RS, Middleton MR and Blesing C: A reply to evidence-based radiation oncology: Oesophagus. Radiother Oncol 94: 387-388, 2010.

5. Berger B and Belka C: Evidence-based radiation oncology: Oesophagus. Radiother Oncol 92: 276-290, 2009.

6. Borghesi S, Hawkins MA and Tait D: Oesophagectomy after definitive chemoradiation in patients with locally advanced oesophageal cancer. Clin Oncol (R Coll Radiol) 20: 221-226, 2008.

7. Li N, Huang D, Lu N and Luo L: Role of the LKB1/AMPK pathway in tumor invasion and metastasis of cancer cells (Review). Oncol Rep 34: 2821-2826, 2015.

8. Gan RY and Li HB: Recent progress on liver kinase B1 (LKB1): Expression, regulation, downstream signaling and cancer suppressive function. Int J Mol Sci 15: 16698-16718, 2014.

9. Zhou W, Zhang J and Marcus AI: LKB1 Tumor suppressor: Therapeutic opportunities knock when LKB1 is inactivated. Genes Dis 1: 64-74, 2014.

10. Momcilovic $M$ and Shackelford DB: Targeting LKB1 in cancer-exposing and exploiting vulnerabilities. Br J Cancer 113: 574-584, 2015.

11. Herter-Sprie GS, Korideck H, Christensen CL, Herter JM, Rhee K, Berbeco RI, Bennett DG, Akbay EA, Kozono D, Mak RH, et al: Image-guided radiotherapy platform using single nodule conditional lung cancer mouse models. Nat Commun 5: $5870,2014$.

12. Saigusa S, Inoue $\mathrm{Y}$, Tanaka K, Toiyama Y, Kawamura M, Okugawa Y, Okigami M, Hiro J, Uchida K, Mohri Y and Kusunoki M: Significant correlation between LKB1 and LGR5 gene expression and the association with poor recurrence-free survival in rectal cancer after preoperative chemoradiotherapy. J Cancer Res Clin Oncol 139: 131-138, 2013.

13. Chen Z, Cheng K, Walton Z, Wang Y, Ebi H, Shimamura T, Liu Y, Tupper T, Ouyang J, Li J, et al: A murine lung cancer co-clinical trial identifies genetic modifiers of therapeutic response. Nature 483: 613-617, 2012.

14. Xia C, Ye F, Hu X, Li Z, Jiang B, Fu Y, Cheng X, Shao Z and Zhuang Z: Liver kinase B1 enhances chemoresistance to gemcitabine in breast cancer MDA-MB-231 cells. Oncol Lett 8: 2086-2092, 2014.

15. Wang YQ, Dai WM, Chu XY, Yang B, Zhao M and Sun Y: Downregulation of LKB1 suppresses Stat 3 activity to promote the proliferation of esophageal carcinoma cells. Mol Med Rep 9: 2400-2404, 2014. 
16. Piao LS, Hur W, Kim TK, Hong SW, Kim SW, Choi JE, Sung PS, Song MJ, Lee BC, Hwang D and Yoon SK: CD133+ liver cancer stem cells modulate radioresistance in human hepatocellular carcinoma. Cancer Lett 315: 129-137, 2012.

17. Paglin S, Hollister T, Delohery T, Hackett N, McMahill M, Sphicas E, Domingo D and Yahalom J: A novel response of cancer cells to radiation involves autophagy and formation of acidic vesicles. Cancer Res 61: 439-444, 2001.

18. Gewirtz DA, Hilliker ML and Wilson EN: Promotion of autophagy as a mechanism for radiation sensitization of breast tumor cells. Radiother Oncol 92: 323-328, 2009.

19. Zois CE and Koukourakis MI: Radiation-induced autophagy in normal and cancer cells: Towards novel cytoprotection and radio-sensitization policies? Autophagy 5: 442-450, 2009

20. Moretti L, Cha YI, Niermann KJ and Lu B: Switch between apoptosis and autophagy: Radiation-induced endoplasmic reticulum stress? Cell Cycle 6: 793-798, 2007.

21. Raja E, Tzavlaki K, Vuilleumier R, Edlund K, Kahata K, Zieba A, Morén A, Watanabe Y, Voytyuk I, Botling J, et al: The protein kinase LKB1 negatively regulates bone morphogenetic protein receptor signaling. Oncotarget 7: 1120-1143, 2016.

22. Just PA, Poncy A, Charawi S, Dahmani R, Traore M, Dumontet T, Drouet V, Dumont F, Gilgenkrantz H, Colnot S, et al: LKB1 and notch pathways interact and control biliary morphogenesis. PLoS One 10: e0145400, 2015.

23. Wang J, Zhang K, Wang J, Wu X, Liu X, Li B, Zhu Y, Yu Y, Cheng Q, Hu Z, et al: Underexpression of LKB1 tumor suppressor is associated with enhanced Wnt signaling and malignant characteristics of human intrahepatic cholangiocarcinoma. Oncotarget 6: 18905-18920, 2015.

24. Lipovka Y and Konhilas JP: AMP-activated protein kinase signalling in cancer and cardiac hypertrophy. Cardiovasc Pharm Open Access 4: pii: 154, 2015.

25. Dasgupta B and Chhipa RR: Evolving lessons on the complex role of AMPK in normal physiology and cancer. Trends Pharmacol Sci 37: 192-206, 2016
26. Sanli T, Rashid A, Liu C, Harding S, Bristow RG, Cutz JC, Singh $\mathrm{G}$, Wright $\mathrm{J}$ and Tsakiridis $\mathrm{T}$ : Ionizing radiation activates AMP-activated kinase (AMPK): A target for radiosensitization of human cancer cells. Int J Radiat Oncol Biol Phys 78: 221-229, 2010.

27. Storozhuk Y, Hopmans SN, Sanli T, Barron C, Tsiani E, Cutz JC, Pond G, Wright J, Singh G and Tsakiridis T: Metformin inhibits growth and enhances radiation response of non-small cell lung cancer (NSCLC) through ATM and AMPK. Br J Cancer 108: 2021-2032, 2013.

28. Muaddi H, Chowdhury S, Vellanki R, Zamiara $\mathrm{P}$ and Koritzinsky M: Contributions of AMPK and p53 dependent signaling to radiation response in the presence of metformin. Radiother Oncol 108: 446-450, 2013.

29. Fasih A, Elbaz HA, Hüttemann M, Konski AA and Zielske SP: Radiosensitization of pancreatic cancer cells by metformin through the AMPK pathway. Radiat Res 182: 50-59, 2014.

30. Shaw RJ, Kosmatka M, Bardeesy N, Hurley RL, Witters LA, DePinho RA and Cantley LC: The tumor suppressor LKB1 kinase directly activates AMP-activated kinase and regulates apoptosis in response to energy stress. Proc Natl Acad Sci USA 101: 3329-3335, 2004.

31. Hardie DG and Hawley SA: AMP-activated protein kinase: The energy charge hypothesis revisited. Bioessays 23: 1112-1119, 2001 . 